\title{
CONSIDERATIONS REGARDING THE REDESIGN OF MANAGEMENT SYSTEM OF AN ORGANIZATION
}

\author{
Alexandru Marius RIZESCU *, George BUCĂŢA ** \\ *“Nicolae Bălcescu" Land Forces Academy, Sibiu, Romania \\ ** "Lucian Blaga" University, Sibiu, Romania \\ mariusrizescu@yahoo.com; george.bucata@yahoo.ro
}

\begin{abstract}
In addition to the current exercise of management functions, managers in any organization, must pursue a permanent adjustment of the management system at the new changes that have occurred, and that may be anticipated in the internal and external environment of the organization. This involves a number of activities of organization change management, components that can be included in what we call redesigning the organization management.
\end{abstract}

Keywords: organizational management, organizational redesign, management system.

\section{Introduction}

A great way to streamline content management through revolutionary management techniques, managerial redesign is determined by the expression of multiple causes:

- manifestation of some major disfunctionalities in management, as well as predominantly empirical character;

- coordination, motivation and communication prevailing deficit and low capacity of response to environmental challenges;

- nationally and internationallyquestionable competence for large numbers of managers of organizations;

- changing passive attitude towards in comparison with the new found out in multiple positions.

For the redesign of the whole management of a company, we recommend the following methods:

- achievement of genuine and complex studies of diagnosis and SWOT analysis which would identify potential managerial and economic viability of the categorical system by outlining objectives (fundamental, specific and individual);

- reengineering of business processes, as well as process redesign support or structural remodelling;

- support, compatibility of managers to organizational management positions they fill;

- redesign, mainly by promoting the use of modern management tools, complex and sophisticated, as well as management on the basis of profit centers or through management improvement;

- objectives of the organizational culture and managerial staff;

- professionalization of managers and management. [3] 


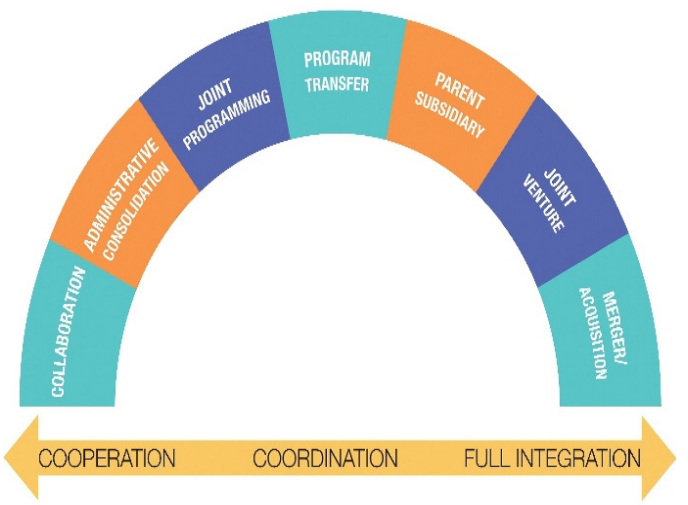

Figure 1: The organizational management redesign

All companies which are being under the pressure from the inside or from outside forces (environment, nationally and internationally), but want consolidating their place on a specific market or to "enter" on other markets use the use a scenario methodology structured into different objectives: structures, people, processes and performance. Managers need to start by analyzing the process of diagnosis, consequence of a strict methodological approach. The most significant information provided by diagnostic study, are: strengths and causes that it generates, the weaknesses and the causes of their occurrence, potential managerial and economic viability and tactical recommendations. [1]

The first starting point, objectives, aim to the objectives of the management and forms a system of objectives resulting from the development of strategies and global policies. The foundation and development of fundamental objectives, specific and individual derivative, the promotion of strategies and realistic policy means a huge change in the view of the companies and the strategy, present and future, approached from a performance point of view. Objectives of commitment means that employees and all the groups from the company should be advised for a pronounced strategic dimension and tactic which is absolutely crucial for normal positioning and market, finally very good benchmarks for assessing the functionality and performance. The objectives must be specific, measurable, affordable, relevant and framed in time. SMART translates requirements through the following: objective must be specific, what managers want to get?, must be measurable, able to be quantified; questions managers must answer in order to check what they did, the objective must be in time. [4]

\section{The Five-Step MBO Process}

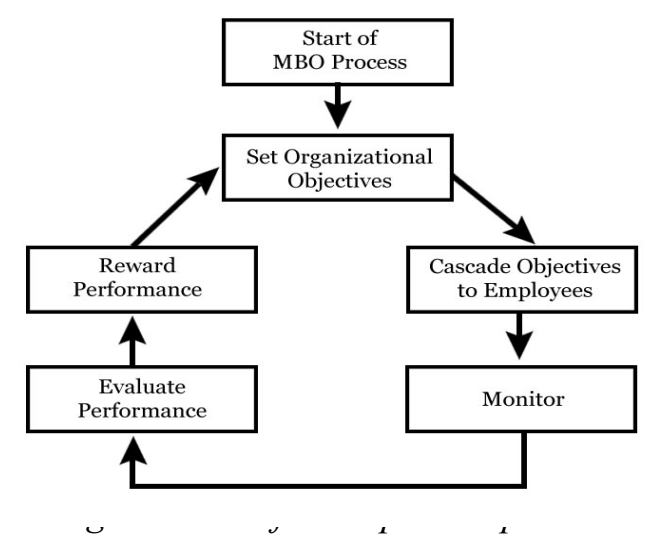

For reaching the objectives, work processes are demanded, with different degrees of aggregation. The size of their business, support processes and management processes are determined by the complexity of the objectives whose achievement is participating directly. Whereas the construction of the system of goals takes place from "top to bottom" it is necessary to achieve their procedural building looms from complex to simple. As such, collaborative process in reshaping the emergence of totally new work processes, development of current work processes, reducing or eliminating others, seeks to ensure full concordance between the goals and processes to develop a "maps of processes", which are pasted into the main activities, generating added value that will be addressed on a priority basis. [2]

Work processes must have an appropriate structural support, organizational structure and positive organizational objectives. That is why, the third step in redesigning the structural management represents and embodies the resizing of the need for the posts and functions, top management and execution, operational and functional 
compartments. The next step is decisive in making sure that there is viability of the company whereas targeting the endowment of management and employees.

Accordingly, the following component is subject to redesign managerial human resources management taking into account that managerial "zone" is extremely dynamic, but also vulnerable, in that the recruitment, selection, employment, evaluation, motivation, improving, promoting and protecting employees and organizational culture. Through human resources quality management ensures the professionalization of the companies:

- managers are involved in the adoption of decisions and justification; information channels transmitted upwards. They respond exclusively to the quality of decisions and, partially, of the effectiveness in their application;

- managers initiate actions needed, exploiting the information transmitted mainly downwards. Also responsible for the effectiveness of the application of the decisions. [6]

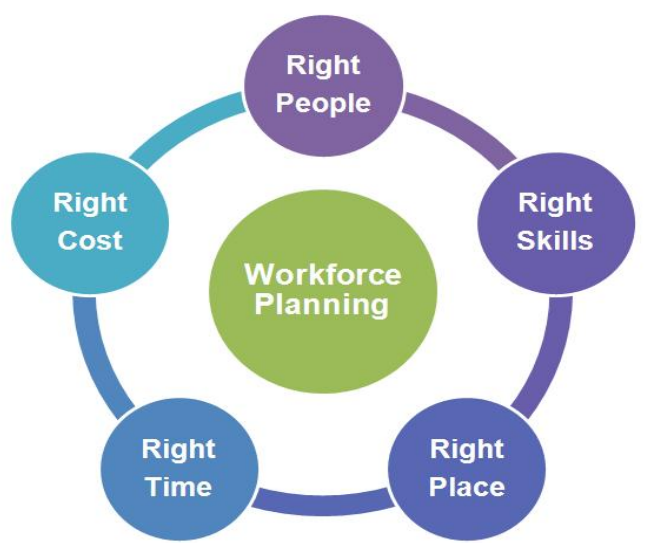

Figure 3: The workforce planning model in management redesign

In regards to the decision-making management, there may be component mutations, resulting in:

- delimitation and dimensioning of the authority or competence of hierarchical levels;

- improving decisions through a more scientific justification by ensuring the operability of the adoption and implementation of appropriate formulation in order to ensure an adequate level of qualitative parameters, but especially for a proper scientific substantiation of the decisions it is necessary the fulfillment of two conditions: on the one hand, ensuring the information needed is in proposer quantity and, especially, in structure and with appropriate quality and, on the other hand, calling the decision-making methods and techniques consistent with the nature of the issues to be resolved (certain decisionmaking uncertain times, risk);

- improving the decisions adopted, typological upside the share strategic and tactical decisions, decisions of risk and uncertainty at higher levels in the hierarchy. [8]

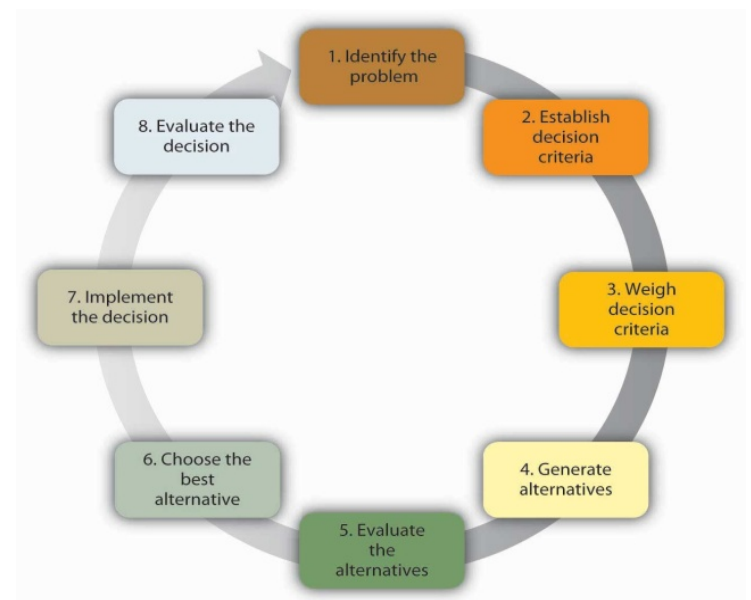

Figure 4: Understanding the decision-making management process

In turn, the redesign of the information involves:

- improving the quality and quantity of the information circulated on the information flows downward and upward;

- streamlining information and statements of the routes on which they are moving;

- increasing computerization of business processes, support and management through the promotion of information systems performance;

- sophistication of information procedures. [12]

In others terms, you can't rewind an solid management process The content served may constitutes the organization, referring to a global diagnostics (recommended in the wider context of the scenario 
methodology) or one or more components of the organizational structure of process times, the situation in which the diagnosis is the first one. [5]

The result of the diagnosis is the diagnostic study. Without it, without capitalizing on content or in relevant decisions and actions, we may not witness major changes in functionality. In this way, it may ensure the multidimensionality of diagnosis in the sense of emphasizing certain aspects of the nature of the economic, managerial, technical, etc. in proportions that would justify their share in the fields subject to investigations. At the same time, a participatory dimension in the elaboration of the pronounced clinical diagnosis and effective through active involvement of managers must be ensured in providing relevant information and in formulating the elements of the essence of the diagnosis.

Stage I: preparing the diagnosis analysis, tracking and securing human conditions, organizational, informational and material required for the effective determination of the diagnostic study.

- the diagnostic team, in compliance with the terms of multidisciplinarity and the origin of it;

- establishing the role of each component of the diagnostic team;

- the type of diagnosis: global and partial;

- scoping subject to investigations in terms of organization or procedural components of its structural fold;

- an indication of the period to be covered;

- establishment of typesetting: instruments, recording and processing of data and information; given the complexity of the diagnosis-especially the global-we recommend the use of simultaneous multiple methods of collection, recording and analysis of data and information.

Stage II: preliminary (investigation) documentation involves the following aspects.

Stage III: analysis of the economic viability of the companies and management, extremely dense, complex, it is necessary to use a diversified analysis instrumentation. [7]

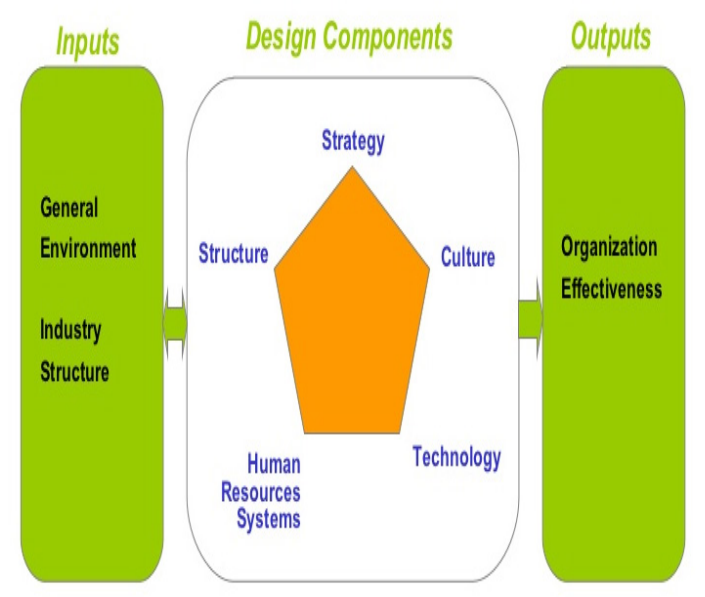

Figure 5: The organizational diagnosis general model

Alltogether, managerial restructuring means reconsidering the following items:

- applying the principle of "the right man at the right time and place" for safe and immediate results;

- concern for the development of knowledge and managerial skills;

- presence of exercising more functions of planning, organizing and control of continuous activities, performance and resources;

- implementation of methods and tools of management: management by objectives, management through budgets, management through projects, management dashboards, so that all the objectives and results to be measurable and controllable, so that they can interviene anytime with corrective measures;

- information system reorganization of the firm, so that managerial team to have access to information and accurate reports, comprehensive and well-structured;

- communication and improve managerial throughout departments;

- revision of the decision-making system, so that the decisions to be well substantiated and that there is a future assessment thereof. [10]

Organizational restructuring means the company analysis and redesign activities in 
line with the new objectives and strategies of the company:

- the company's organizational structure, review in terms of hierarchical levels, responsibility centres, productive centres and support centres;

- redesigning the number and personal policy, wage policy;

- development of controlling departments for monitoring, analyzing, assessing financial and commercial performance of the business and the granting of any information management team in order to elaborate strategies and policies of the company; [11]

The financial restructuring is aimed at maintaining profitability and business financial equilibrium, through continuous monitoring of revenues and costs and receipts and payments with third parties. To achieve this objective, the following should be considered:

- measures and identification/elimination of areas that generate cash losses: production, sales support; range of products or services;

- reducing costs and optimizing their structure according to the volume and value of sales;

- development and follow-up monthly income and expense budget-level company and centre of responsibility for compliance with the limits established by the budget and premises for anticipating and reducing losses;
- elaboration and monitoring of monthly and weekly budgets of receipts and payments for the foresight and ensure funding needs for continuation of the activity of the company;

- investment policy review and bank financing;

- funding policy review: credit commercial client and supplier credit;

- designing and monitoring key indicators of financial and economic analysis of profitability, liquidity a system of implementation of financial management dashboards for piloting the efficiency of the business. [9]

\section{Conclusions}

The restructuring and redesign of management are two important ways organizations change, recommended along with privatization, reorganization, improvement, modernization, rationalization. The first of these, restructuring, became a "fashion" of the past few years, even though the results achieved as a result of such an approach cannot be catalogued as being favorable. In terms of management, redesign its retrieval in the category of managerial change is only theoretical, so due to its content without knowing the exact methodology, as well as due to shortage of facts indicating the implications for managerial and economic performance of the organization in general, and in particular economic operators.

\section{References}

[1] Arijit Sikdar Jayashree Payyazhi, (2014), “A process model of managing organizational change during business process redesign", Business Process Management Journal, Vol. 20 Iss 6 pp. 971 - 998;

[2] Doebeli, G., Fisher, R., Gapp, R. and Sanzogni, L. (2011), "Using BPM governance to align systems and practice", Business Process Management Journal, Vol. 17 No. 2, pp. 184-202;

[3] Kates A, Galbraith JR. 2010. Designing Your Organization: Using the Star Model to Solve 5 Critical Design Challenges. Jossey-Bass, San Francisco, CA.;

[4] Kohlbacher, M. (2010), "The effects of process orientation: a literature review", Business Process Management Journal, Vol. 16 No. 1, pp. 135-152; 
[5] Manyika J, Chui M, Brown B, Bughin J, Dobbs B, Roxburgh C, Byers AH. 2011. Big data: The next frontier for innovation, competition, and productivity. McKinsey Global Institute;

[6] Nissen ME, Burton RM. 2011. Designing organizations for dynamic fit: System stability, maneuverability and opportunity loss. IEEE Transactions on Systems, Man and Cybernetics, Part A 41(4): 418-433;

[7] Nicolescu, Ovidiu, and Lester Lloyd-Reason. "Contributions to Redesigning the Management System in Medium-Sized Enterprises", Challenges Performances and Tendencies in Organisation Management, 2016;

[8] Schynsa, B. y Schilling, J. (2014). How bad are the effects of bad leaders? A metaanalysis of destructive leadership and its outcomes. The Leadership Quarterly, 24(1), 138-158;

[9] Segatto, M., de Padua, S.I.D. and Martinelli, D.P. (2013), "Business process management: a systemic approach?”, Business Process Management Journal, Vol. 19 No. 4, pp. 698-714;

[10] Siriram, R. (2012), "A soft and hard systems approach to business process management", Systems Research and Behavioral Science, Vol. 29 No. 1, pp. 87-100;

[11] Reid,Wilbur A., I.,II, West, G. R. B., Winston, B. E. y Wood, J. (2014). An instrument to measure level 5 leadership. Journal of Leadership Studies, 8(1), 17-32;

[12] Zhao X, Liu C. 2010. Steering dynamic collaborations between business processes. IEEE Transactions on Systems, Man and Cybernetics, Part A, 40(4): 743-757. 